\title{
Foundation of a unified physics
}

\author{
Ivanhoe B. Pestov \\ ${ }^{1}$ Joint institute for nuclear research, 141980, Dubna, Moscow Region, Russia
}

\begin{abstract}
Since searches of new physics is not successful till now we need to have new approaches to the fundamental physics as a whole. In this report we exhibit new system of fundamental equations of physics (derived from the first principle) which gives rise to new physics and provides a basis for comparison with current one.
\end{abstract}

\section{Introduction}

To formulate new unified approach and understand why nature is just the way it is we abstract away from the known concepts and laws (a writer must not share your point of view) and put forward the grand relation: fundamental physical ideas, symmetries and equations are tightly connected with the idea of space and, hence, they have the geometrical origin and are formulated in the form which is completely independent of any outer and a priori conditions (everything is the concept of space and the concept of space is everything). Thus, the radically new idea of a unified physics is the most possible generality and the outlined first principle. The presented model is the only one unique logically possible physical theory since it gives the solution of the most difficult and long-standing problem in the history of science - the problem of time. When we discover connections of time with other objects, the reason of the existence of time becomes clear. The main body of references:[1]-[8].

\section{General characteristic of fields and time}

The most general realization of the idea of space is the concept of physical manifold. The physical manifold is a smooth manifold plus its connections with physical fields that transfer energy and momentum. The geometrical structure of the physical manifold (points, curves, congruences of curves, families of curves) determines a very restricted set of really geometrical quantities and along with that geometrical internal symmetry that makes these quantities variable and forms from them the fundamental physical fields: the positively defined Riemann metric $g_{i j}$ which is the potential of the gravitational field providing connection of the physical fields with the physical manifold (it is characterized by the reparameterization invariance);

the linear (affine) connection $P^{i}{ }_{j k}$ which is the potential of the generalized electromagnetic field ( the group of geometrical internal symmetry is a general linear group);

the scalar and covariant vector fields, and antisymmetric covariant tensor fields which are connected by the geometrical internal symmetry (spin symmetry) into the real spin field

$$
\mathbf{A}=\left(a, a_{i}, a_{i j}, a_{i j k}, a_{i j k l}\right), \quad i, j, k, l=1,2,3,4 .
$$


The intrinsic space-time structure of the physical manifold is the temporal field (the natural clock) and bilateral symmetry defined by the arrow of time. The temporal field with respect to the coordinate system $u^{1}, u^{2}, u^{3}, u^{4}$ is denoted by $f(u)=f\left(u^{1}, u^{2}, u^{3}, u^{4}\right)$. The reading $t$ of the natural clock is defined by the formula $t=f(u)=f\left(u^{1}, u^{2}, u^{3}, u^{4}\right)$ for any point of the physical manifold. The two events at the points $u^{1}, u^{2}, u^{3}, u^{4}$ and $v^{1}, v^{2}, v^{3}, v^{4}$ go on concurrently if $f\left(u^{1}, u^{2}, u^{3}, u^{4}\right)=f\left(v^{1}, v^{2}, v^{3}, v^{4}\right)$. The gradient of the temporal field (the arrow of time) is the vector field $\mathbf{t}$ with the components $t^{i}=(\nabla f)^{i}=$ $g^{i j} \partial_{j} f=g^{i j} t_{j}$, where $g^{i j}$ are the contravariant components of the Riemann metric $g_{i j}$. The construction of natural clock is defined by the fundamental equation :

$$
D_{\mathbf{t}} f=(\nabla f)^{2}=g^{i j} \frac{\partial f}{\partial u^{j}} \frac{\partial f}{\partial u^{j}}=1,
$$

which means that time flows equably. This equation has a general solution and a special solution known as the function of geodesic distance.

The arrow of time defines the fundamental discrete internal symmetry- bilateral symmetry. A pair of vector fields $\mathbf{v}$ and $\overline{\mathbf{v}}$ has bilateral symmetry if the sum of these fields is collinear to the gradient of a temporal field and their difference is orthogonal to it, $\overline{\mathbf{v}}+\mathbf{v}=\lambda \mathbf{t}, \quad(\overline{\mathbf{v}}, \mathbf{t})=(\mathbf{v}, \mathbf{t})$, where $(\mathbf{v}, \mathbf{w})=g_{i j} v^{i} w^{j}=v^{i} w_{i}$ is a scalar product. The bilateral symmetry may be represented as a linear transformation (reflection) $\bar{v}^{i}=R_{j}^{i} v^{j}$, where $R_{j}^{i}=2 t^{i} t_{j}-\delta_{j}^{i}$.

The bilateral symmetry defines the auxiliary metric $\bar{g}_{i j}=2 t_{i} t_{j}-g_{i j}=g_{i k} R_{j}^{k}, \quad \bar{g}^{i j}=2 t^{i} t^{j}-g^{i j}$, which defines time evolution of the physical fields.

In the geometrical (coordinate independent) form the time reversal invariance means that the theory is invariant with respect to the transformations $T: t^{i} \rightarrow-t^{i}$.

\section{Equations of Gravidynamics}

The dynamical equations of the gravitational field and the law of energy conservation are represented in this section.

We denote

$$
\Gamma_{j k}^{i}=\frac{1}{2} g^{i l}\left(\partial_{j} g_{k l}+\partial_{k} g_{j l}-\partial_{l} g_{j k}\right), \quad R_{i j k}^{l}=\partial_{i} \Gamma_{j k}^{l}-\partial_{j} \Gamma_{i k}^{l}+\Gamma_{i m}^{l} \Gamma_{j k}^{m}-\Gamma_{j m}^{l} \Gamma_{i k}^{m}
$$

and get the Ricci tensor $R_{j k}=R_{l j k}^{l}$ and covariant derivative $\nabla_{i}$ with respect to $\Gamma_{j k}^{i}$. With this we introduce momentum of the gravitational field

$$
P_{j}^{i}=\frac{1}{2} g^{i k} D_{\mathbf{t}} g_{j k}=\frac{1}{2} g^{i k}\left(\nabla_{j} t_{k}+\nabla_{k} t_{j}\right)=g^{i k} \nabla_{j} t_{k}=\nabla_{j} t^{i}
$$

where $D_{\mathbf{t}}$ is the Lie derivative along the arrow of time. The stress tensor of the gravitational field can be written in the following form:

$$
S_{j}^{i}=h_{k}^{i} R_{l}^{k} h_{j}^{l}+D_{\mathbf{t}} P_{j}^{i}+\varphi P_{j}^{i}
$$

where $\varphi=\nabla_{i} t^{i}$ and $h_{j}^{i}=\delta_{j}^{i}-t_{j} t^{i}$. For a density of kinetic and potential energy of the gravitational field we have the following expressions:

$$
T=\frac{1}{2}\left(P_{i}^{i}\right)^{2}-\frac{1}{2} P_{j}^{i} P_{i}^{j}=\frac{1}{2}(\operatorname{Tr} P)^{2}-\frac{1}{2} \operatorname{Tr}\left(P^{2}\right), \quad U=\frac{1}{2} S=\frac{1}{2} S_{l}^{l} .
$$


The first group of equations of Gravidynamics reads

$$
D_{\mathbf{t}} P_{j}^{i}+\varphi P_{j}^{i}+S_{j}^{i}+N_{j}^{i}=\frac{1}{2} \varepsilon h_{j}^{i},
$$

where $N_{j}^{i}$ is the stress tensor of the generalized electromagnetic field and spin field, $\varepsilon$ is the energy density of the gravitational field and other fields $\varepsilon=\varepsilon_{\mathrm{g}}+\varepsilon_{\mathrm{m}}$. The second group of equations of Gravidynamics reads

$$
\mathrm{G}_{j}=\Pi_{j}
$$

where

$$
\mathrm{G}_{j}=h_{j}^{i}\left(\nabla_{l} P_{i}^{l}-\partial_{i} P_{l}^{l}\right)
$$

is the energy flow vector of the gravitational field and $\Pi_{i}$ is the energy flow vector of the other fields.

The law of energy conservation reads

$$
D_{\mathbf{t}}(\sqrt{g} \varepsilon)=0, \quad \varepsilon=\varepsilon_{\mathbf{g}}+\varepsilon_{\mathbf{m}}=T+U+\varepsilon_{\mathbf{m}} .
$$

Since the temporal field enters into the Lagrangians of the physical fields in the form of the gradient of the scalar field $t_{i}=\partial_{i} f(u)$, the laws of a unified physics are invariant with respect to transformations of the form $f(u) \Rightarrow f(u)+a$, where $a$ is a constant. This is internal symmetry that defines the law of energy conservation as a fundamental physical law of the nature which is true in all cases.

\section{Equations of Generalized Electromagnetic Field}

Here we establish dynamical equations of the generalized electromagnetic field (shortly gef) which are defined by gefsymmetry and describe a new form of matter which interacts only gravitationally and by this reason can be identified with the so called dark matter.

The potential of generalized electromagnetic field $P_{j k}^{i}$ is characterized by the internal symmetry $G L(4, \mathbf{R})$ with the law of transformation $\bar{P}_{j k}^{i}=U_{m}^{i} P_{j n}^{m} V_{k}^{n}+U_{m}^{i} \partial_{j} V_{k}^{m}$, where $V_{j}^{i}$ are the components of the operator $U^{-1}$ inverse to the operator $U, U_{k}^{i} V_{j}^{k}=\delta_{j}^{i}$. For brevity, we use the matrix notation $\mathbf{U}=$ $\left(U_{l}^{k}\right), \quad \mathbf{P}_{i}=\left(P_{i l}^{k}\right), \quad \mathbf{E}=\left(\delta_{l}^{k}\right), \quad \mathbf{H}_{i j}=\left(H_{i j l}{ }^{k}\right), \quad \operatorname{Tr} \mathbf{U}=U_{k}^{k}$. The transformations of gefsymmetry take the form $\overline{\mathbf{P}}_{i}=\mathbf{U} \mathbf{P}_{i} \mathbf{U}^{-1}+\mathbf{U} \partial_{i} \mathbf{U}^{-1}=\mathbf{P}_{i}+\mathbf{U D}_{i} \mathbf{U}^{-1}$, where $\mathrm{D}_{i}$ is the natural differential operator associated with gefsymmetry only $\mathrm{D}_{i} \mathbf{U}=\partial_{i} \mathbf{U}+\mathbf{P}_{i} \mathbf{U}-\mathbf{U} \mathbf{P}_{i}=\partial_{i} \mathbf{U}+\left[\mathbf{P}_{i}, \mathbf{U}\right]$.

The Riemann tensor $\mathbf{B}_{i j}=\partial_{i} \mathbf{P}_{j}-\partial_{j} \mathbf{P}_{i}+\left[\mathbf{P}_{i}, \mathbf{P}_{j}\right]$ of $P_{j k}^{i}$ is reducible with respect to the transformations $\overline{\mathbf{B}}_{i j}=\mathbf{U B}_{i j} \mathbf{U}^{-1}$, since $\mathbf{B}_{i j}=\left(\mathbf{B}_{i j}-\frac{1}{4} \operatorname{Tr}\left(\mathbf{B}_{i j}\right) \mathbf{E}\right)+\frac{1}{4} \operatorname{Tr}\left(\mathbf{B}_{i j}\right) \mathbf{E}$. Hence, the strength tensor of the generalized electromagnetic field is given by the formula

$$
\mathbf{H}_{i j}=\mathbf{B}_{i j}-\frac{1}{4} \operatorname{Tr}\left(\mathbf{B}_{i j}\right) \mathbf{E}, \quad \operatorname{Tr}\left(\mathbf{H}_{i j}\right)=0
$$

and the singlet state of the gef defines the tensor of the electromagnetic field $F_{i j}=\operatorname{Tr}\left(\mathbf{B}_{i j}\right)$.

The ground state of gef is defined by the equation $\mathbf{B}_{i j}=0$. We give a general solution of this equation. Let's consider four linear independent vector fields $E_{\mu}^{i}$ and one can construct purely algebraic components of the four covector fields $E_{i}^{\mu}$, so that $E_{\mu}^{i} E_{j}^{\mu}=\delta_{j}^{i}$ holds valid. Setting $P_{j k}^{i}=L_{j k}^{i}$, where $L_{j k}^{i}=E_{\mu}^{i} \partial_{j} E_{k}^{\mu}$, we get a general solution of the equation in question. Let us introduce a tensor field $Q_{j k}^{i}=P_{j k}^{i}-L_{j k}^{i}$ and an irreducible deviation tensor.

$$
T_{j k}^{i}=Q_{j k}^{i}-\frac{1}{4} Q_{j l}^{l} \delta_{k}^{i}, \quad \mathbf{T}_{j}=\mathbf{Q}_{j}-\frac{1}{4} \operatorname{Tr}\left(\mathbf{Q}_{j}\right) \mathbf{E} .
$$


Furthermore, we introduce electric strength and magnetic strength of the generalized electromagnetic field by the direct and inverse mapping

$$
\mathbf{J}_{i}=t^{k} \mathbf{H}_{i k}, \quad \mathbf{M}_{i}=\frac{1}{2} e_{i k j l} t^{k} \mathbf{H}^{j l}=t^{k} \dot{H}_{i k}^{*}, \quad \mathbf{H}_{i k}=-t_{i} \mathbf{J}_{k}+t_{k} \mathbf{J}_{i}-e_{i k j l} t^{j} \mathbf{M}^{l} .
$$

The Lagrangian of gefdynamics takes the form

$$
\mathcal{L}_{P}=-\frac{1}{4} \operatorname{Tr}\left(\mathbf{H}_{i j} \widetilde{\mathbf{H}}^{i j}\right)-\frac{\mu^{2}}{2} \operatorname{Tr}\left(\mathbf{T}_{i} \widetilde{\mathbf{T}}^{i}\right)
$$

where $\mu$ is a constant of dimension of $\mathrm{cm}^{-1}, \widetilde{\mathbf{H}}^{i j}=\bar{g}^{i k} \bar{g}^{j l} \mathbf{H}_{k l}=2 t^{i} \mathbf{J}^{j}-2 t^{j} \mathbf{J}^{i}+\mathbf{H}^{i j}, \quad \widetilde{\mathbf{T}}^{i}=\bar{g}^{i k} \mathbf{T}_{k}$.

The first pair of equations of gefdynamics can be represented in the following form:

$$
t^{k} \mathrm{D}_{k} \mathbf{M}^{i}-\mathbf{M}^{k} \partial_{k} t^{i}+\varphi \mathbf{M}^{i}=-e^{i j k l} t_{j} \mathrm{D}_{k} \mathbf{J}_{l}, \quad \frac{1}{\sqrt{g}} \mathrm{D}_{i}\left(\sqrt{g} \mathbf{M}^{i}\right)=0
$$

where $\varphi=\nabla_{i} t^{i}$

The second pair of equations of gefdynamics reads

$$
t^{k} D_{k} \mathbf{J}^{i}-\mathbf{J}^{k} \partial_{k} t^{i}+\varphi \mathbf{J}^{i}=e^{i j k l} t_{j} \mathrm{D}_{k} \mathbf{M}_{l}+\mu^{2} \mathbf{S}^{i}, \quad \frac{1}{\sqrt{g}} \mathrm{D}_{i}\left(\sqrt{g} \mathbf{J}^{i}\right)=\mu^{2} \mathbf{S}
$$

where $\mathbf{S}_{i}=h_{i}^{j} \mathbf{T}_{j}, \quad \mathbf{S}=t^{k} \mathbf{T}_{k}$.

Since $\operatorname{Tr}\left(\mathbf{J}_{i}\right)=\operatorname{Tr}\left(\mathbf{M}_{i}\right)=\operatorname{Tr}\left(\mathbf{T}_{i}\right)=0$, the system of equations in question is simultaneous. This system of equations describes a new form of matter which is the structure element of the unified physics and has the characteristics of an entity known from the observations as Dark Matter.

\section{Equations of Spindynamics}

In a Unified Physics the Spindynamics includes the region of physical phenomena which are now investigated in the Standard Model. Thus, spin symmetry is geometrical internal symmetry which provides new and complete understanding of spin and, hence, theory of elementary particles as well. Here we present equations of Spindynamics and introduce operators of electrical charge and neutrino charge and discuss some questions connected with this innovation.

The spin symmetry group is a general linear group $G L(16, \mathbf{R})$ that acts in the linear space that represents the spin field. We constructed a natural general covariant basis in the Lie algebra $g l\left(2^{n}, \mathbf{R}\right)$ of $G L\left(2^{n}, \mathbf{R}\right)$ and describe spin as a bipolar structure on the group of spin symmetry $G L\left(2^{n}, \mathbf{R}\right)$. The bipolar structure means that the two dual sets of commuting operators exist which define the Lie algebra of two dual groups $S$ and $\widetilde{S}$. This result expresses in the mathematical form that the spin field can be put in correspondence to the physical top and that this field is the space of the two-valued representation of the dual groups $S$ and $\widetilde{S}$.

To write Lagrangians of Spindynamics we define the positive definite scalar product and the auxiliary scalar product in the linear space in question as follows:

$$
(\mathbf{A} \mid \mathbf{B})=\sum_{p=0}^{4} \frac{1}{p !} a_{i_{1} \cdots i_{p}} b_{j_{1} \cdots j_{p}} g^{i_{1} j_{1}} \cdots g^{i_{p} j_{p}}, \quad\langle\mathbf{A} \mid \mathbf{B}\rangle=\sum_{p=0}^{4} \frac{1}{p !} a_{i_{1} \cdots i_{p}} b_{j_{1} \cdots j_{p}} \bar{g}^{i_{1} j_{1}} \cdots \bar{g}^{i_{p} j_{p}} .
$$

The relation $\langle\mathbf{A} \mid \mathbf{B}\rangle=(\mathbf{A} \mid \mathrm{R} \mathbf{B})$ holds valid for these two scalar products, where $\mathrm{R}=-Q_{\mathbf{t}} \widetilde{Q}_{\mathbf{t}}=Q_{-\mathbf{t}} \widetilde{Q}_{\mathbf{t}}=$ $Q_{\mathrm{t}} \widetilde{Q}_{-\mathrm{t}}$ and

$$
Q_{\mathbf{t}}: \bar{a}_{i_{1} \cdots i_{p}}=p t_{\left[i_{1}\right.} a_{\left.i_{2} \cdots i_{p}\right]}-t^{k} a_{k i_{1} \cdots i_{p}}, \quad \widetilde{Q}_{\mathbf{t}}: \bar{a}_{i_{1} \cdots i_{p}}=(-1)^{p+1}\left(p t_{\left[i_{1}\right.} a_{\left.i_{2} \cdots i_{p}\right]}+t^{k} a_{k i_{1} \cdots i_{p}}\right) .
$$


The square brackets $[\cdots]$ denote the process of alternation, $t^{i}=g^{i j} t_{j}$ and $p=0,1,2,3,4$. The operator $\mathrm{R}$ gives the straightforward representation of the bilateral symmetry and shows that this representation opens a new possibility to interpret the bilateral symmetry in the case of the spin field.

The Lagrangian of Spindynamics associated with this new representation of bilateral symmetry can be written only for the complex spin field and has the form:

$$
\mathcal{L}_{\mathbf{t}}=-\frac{i}{2}\left\langle\widetilde{Q}_{\mathbf{t}} \stackrel{*}{\Psi} \mid \Pi \boldsymbol{\Psi}\right\rangle+\frac{i}{2}\left\langle\widetilde{Q}_{\mathbf{t}} \boldsymbol{\Psi} \mid \Pi \stackrel{*}{\boldsymbol{\Psi}}\right\rangle+i m\left\langle\widetilde{Q}_{\mathbf{t}} \stackrel{*}{\boldsymbol{\Psi}} \mid \boldsymbol{\Psi}\right\rangle
$$

It is not invariant with respect to time reversal $T: \quad t_{i} \rightarrow-t_{i}$, since $\mathcal{L}_{\mathbf{- t}}=-\mathcal{L}_{\mathbf{t}}$. The main differential operator of Spindynamics $\Pi$ is defined by the new geometrical object, fundamental connection of Spindynamics

$$
\bar{\Gamma}_{j k}^{i}=\Gamma_{j k}^{i}+t^{i} \nabla_{j} t_{k}-t_{k} \nabla_{j} t^{i}
$$

which is characterized by the equations $\bar{\nabla}_{i} t_{j}=0, \quad \bar{\nabla}_{i} g_{j k}=0, \quad \bar{\nabla}_{i} g^{j k}=0$, where $\bar{\nabla}_{i}$ is the covariant derivative with respect to the connection (6). The operator $\Pi$ is defined as follows:

$$
(\Pi \mathbf{A})_{i_{1} \cdots i_{p}}=p \bar{\nabla}_{\left[i_{1}\right.} a_{\left.i_{2} \cdots i_{p}\right]}+\bar{\nabla}^{k} a_{k i_{1} \cdots i_{p}}-2 t^{k} \bar{\nabla}_{\mathbf{t}} a_{k i_{1} \cdots i_{p}} .
$$

The Lagrangian of Spindynamics associated with the customary representation of bilateral symmetry can be written for the real spin field and takes the following form:

$$
\mathcal{L}_{\mathbf{t}}=\frac{1}{2}\langle\mathbf{A} \mid \Pi \mathbf{A}\rangle-\frac{m}{2}\langle\mathbf{A} \mid \mathbf{A}\rangle .
$$

It is invariant with respect to the time reversal.

Equations for the free complex and real spin fields have the same form :

$$
\Pi \Psi+\frac{1}{2} \varphi Q_{\mathbf{t}} \boldsymbol{\Psi}=m \boldsymbol{\Psi}, \quad \Pi \mathbf{A}+\frac{1}{2} \varphi Q_{\mathbf{t}} \mathbf{A}=m \mathbf{A},
$$

where $\varphi=\nabla_{i} t^{i}$, but there is a principal difference between the Lagrangians (5) and (7).

Since $\Pi \widetilde{Q}_{\mathbf{t}}-\widetilde{Q}_{\mathbf{t}} \Pi=0, \quad\left\langle\widetilde{Q}_{\mathbf{t}} \mathbf{A} \mid \mathbf{B}\right\rangle=-\left\langle\mathbf{A} \mid \widetilde{Q}_{\mathbf{t}} \mathbf{B}\right\rangle$, the Lagrangian (7) is invariant with respect to the transformations $\overline{\mathbf{A}}=\exp \left(\alpha \widetilde{Q}_{\mathbf{t}}\right) \mathbf{A}$. For the density of the corresponding conserved current $C^{k}$ one can find the expression $\rho=t_{i} C^{i}=\left\langle Q_{\mathbf{t}} \mathbf{A} \mid \widetilde{Q}_{\mathbf{t}} \mathbf{A}\right\rangle=(\mathbf{A} \mid \mathbf{A})$ which shows that there is a natural probability measure in the space of solution of equation (8) for the real spin field. This fundamental result gives us an opportunity to equate the operator $\widetilde{Q}_{\mathbf{t}}$ with an operator of the electrical charge $\widetilde{Q}_{\mathbf{t}}=Q_{e}$.

The operator of the neutrino charge $Q_{v}$ should be connected with the parity nonconservation and, hence, it is defined by the orientation of the physical manifold. The orientation is the antisymmetrical tensor $e_{i j k l}$ ("element of volume") normalized as $e_{1234}=\sqrt{g}$, where $g=\operatorname{Det}\left(g_{i j}\right)$.

We define the operator $\widetilde{H}$ in the component form

$$
(\widetilde{H} \mathbf{A})_{i_{1} \cdots i_{p}}=\frac{1}{(4-p) !}(-1)^{\frac{p(p+1)}{2}} e_{i_{1} \cdots i_{p} j_{1} \ldots j_{4-p}} a^{j_{1} \ldots j_{4-p}}
$$

and derive the following relations:

$$
\widetilde{H}^{2}=E, \quad \widetilde{H} \widetilde{Q}_{\mathbf{t}}+\widetilde{Q}_{\mathbf{t}} \widetilde{H}=0, \quad \widetilde{H} Q_{\mathbf{t}}=Q_{\mathbf{t}} \widetilde{H}=0 .
$$

The operator of the neutrino charge $Q_{v}$ takes the form $Q_{v}=\widetilde{Q_{\mathbf{t}}} \widetilde{H} Q_{\mathbf{t}}$. The operators $Q_{e}$ and $Q_{v}$ anticommute

$$
Q_{e} Q_{v}+Q_{v} Q_{e}=0, \quad Q_{e}^{2}=Q_{v}^{2}=-E
$$


The operators $Q_{e}, Q_{v}$ and $Q_{e v}=Q_{e} Q_{v}$ define the representation of $S U(2)$ group and the quaternion structure.

The Lagrangian of Spindynamics (5) is invariant with respect to the transformations

$$
\overline{\boldsymbol{\Psi}}=\exp \left(\alpha Q_{e}\right) \boldsymbol{\Psi}, \quad \overline{\boldsymbol{\Psi}}=\exp \left(\beta Q_{v}\right) \boldsymbol{\Psi}, \quad \overline{\boldsymbol{\Psi}}=\exp \left(\gamma Q_{e v}\right) \boldsymbol{\Psi}
$$

but it is remarkable that the Lagrangian (7) is invariant only with respect to the transformation generated by the operator of the electric charge. We see that in one case the spin field carries only the electrical charge but in the second case it carries the electrical, neutrino and pseudo-charge. In both cases the spin field carries the internal spin which is generated by the geometrical internal symmetry defined by the antisymmetrical tensor field $S_{i j}$ obeying the equation $\bar{\nabla}_{i} S_{j k}=0$. We have no possibility to discuss here this important symmetry in detail.

\section{Conclusion}

Let us mention some evidences of the New Physics. Of course, the principal result is the dynamical equations of the gravitational field, the spin field and the generalized electromagnetic field which form the nucleus of the Unified Physics as a system and this system will reveal its internal content in the process of its exploitation.

It is discovered that in the Universe there are two space-time structures (the two natural clocks). One of them is defined by a general solution of the equation for temporal field (1). Another solution and a new space-time structure emerge as a special solution of this equation and corresponds to the rotating matter. The new space-time structure naturally explains the confinement and the baryon number conservation. Indeed, we cannot invoke the artificial concept of force to explain the confinement because for any force there is a more powerful one. But the new space-time structure is something else again. The baryon number conservation simply expresses in the symbolic form the existence of the second space-time structure and nothing else. The quark - lepton symmetry means that leptons live in the area of the usual space-time structure and quarks live in the area of the new one.

Spindynamics can be considered as a new purely rational realization of the Standard Model derived from the first principle only. The observed generations of quarks and leptons are different states of the complex spin field and the number of these generations equals four. The spin field describes the physical entity retaining the elementarity and is considered to be characterized solely by equations (8), the electrical charge and the internal spin in one case and the pseudocharge, electric charge, neutrino charge and the internal spin in the other case.

The problem of Dark Energy is solved. Dark Energy is simply the energy of the gravitational field. The gravitational field is physical one.

The problem of Dark Matter is solved. The Dark Matter is heavy light that interacts only gravitationally. Heavy photons are quants of the generalized electromagnetic field. Massless photons represent a singlet state of this field.

The most general and universal law of energy conservation is discovered it is valid in all cases and is an opportunity to be a new contributor of energy.

\section{References}

[1] I.B. Pestov. In: Horizons in World Physics, Vol.248, Ed. by A. Reimer, (Nova Science, New York, 2005), 1. Preprint No.E2-2004-105 JINR (Dubna, 2004). gr-qc/0507131

[2] I.B. Pestov, New concept of time and gravity, In: Symmetries and Intergrable Systems, Vol. II, Ed. by A.N. Sissakian. Preprint No. E2-96-424, JINR (Dubna, 1996) 
[3] I.B. Pestov, J.Phys. A: Math.Gen. 33, 3027 (2000)

[4] I.B. Pestov. In: Dark Matter: New Research, Ed. by Val Blain (Nova Science, New York 2006), 77. Preprint No. E2-2005-51, JINR (Dubna, 2005). gr-qc/0412096

[5] I.B. Pestov. Physics of Particles and Nuclei 44(3), 442 (2013)

[6] I.B. Pestov, Theor. and Math. Phys. 34(1), 48 (1978)

[7] I.B. Pestov, Physics of Atomic Nuclei 74(7), 1084 (2011). Preprint No. E2-2008-93, JINR (Dubna, 2008)

[8] I.B. Pestov, J.Phys.Math. 6, 135 (2015). doi: 10.4172/2090-0902.1000135 